SIT-HEP/TM-10

\title{
Kaluza-Klein modes in hybrid inflation
}

\author{
Tomohiro Matsuda f \\ Laboratory of Physics, Saitama Institute of Technology, \\ Fusaiji, Okabe-machi, Saitama 369-0293, Japan
}

\begin{abstract}
When one constructs specific models with the fundamental scale as low as the $\mathrm{TeV}$ scale, there arise many difficulties. In this paper we examine the hybrid inflation due to bulk scalar fields, which has been proposed to solve the problem of fine-tuning in producing density perturbations. We find that the Kaluza-Klein modes play significant roles, which enhance the speed of the phase transition and alter the reheating process. We also argue that a lower bound must be put to the fundamental scale, in order to construct the successful hybrid inflation due to the bulk scalar fields.
\end{abstract}

\footnotetext{
${ }^{1}$ matsuda@sit.ac.jp
} 


\section{Introduction}

In spite of the great success in the quantum field theory, there is still no consistent scenario in which the quantum gravity is included. The most promising scenario in this direction would be the string theory, where the consistency is ensured by the requirement of additional dimensions. Initially the sizes of extra dimensions had been assumed to be as small as $M_{p}^{-1}$, but later it has been observed that there is no reason to believe such a tiny compactification radius [1]. The idea of the large extra dimension may solve or weaken the hierarchy problem. Denoting the volume of the $n$-dimensional compact space by $V_{n}$, the observed Planck mass is obtained by the relation $M_{p}^{2}=M_{*}^{n+2} V_{n}$, where $M_{*}$ denotes the fundamental scale of gravity. If one assumes more than two extra dimensions, $M_{*}$ may be close to the $\mathrm{TeV}$ scale without conflicting any observable bounds.

Although this new idea inspired creativity in many physicists to lead them to a new paradigm of phenomenology, a drastic modification is required for the conventional cosmological scenarios. Models of inflation and baryogenesis[2] are especially sensitive to this low fundamental scale. To avoid extreme fine tuning, we should reconstruct the conventional scenarios of the standard cosmology. This requires inclusion of novel ideas that are quite different from the conventional one that was used for the models with large fundamental scale $M_{*} \sim M_{p}$, where $M_{p}$ denotes the Planck scale. This makes it difficult to construct a specific model for the early evolution of the Universe. For example, if one puts the inflaton fields on the brane, their masses are required to be unnaturally small[3]. On the other hand, in generic cases the mass of the inflaton is bounded from below to achieve successful reheating. Thus it seems quite difficult to construct a model for successful inflation driven by a field on the brane.

A way to avoid these difficulties is put forward by Arkani-Hamed et al. [4], where inflation is assumed to occur before the stabilization of the internal dimensions. In this case, however, the late oscillation of the radion is a problem, which will be solved by the second weak inflation.

One can find other ways to solve problems of inflation in models with large extra dimensions. Due to some dynamical mechanisms, the extra dimension may be stabilized before the Universe exited from inflation. If the stabilization of the internal dimensions 
occurred before the end of inflation, inflation cannot be induced by the fields on the brane, since their energy densities are highly suppressed. In this case one may use the bulk field rather than a field on the brane[5, 6].

One may find another interesting possibility, "brane inflation" [9], which uses the interbrane distance as the inflaton.

In this paper we focus our attention to the second possibility, where the bulk field drives hybrid inflation after the radion stabilization.

We first make a brief review of the hybrid bulk field inflation, then we will examine the effect of the Kaluza-Klein modes.[] In ref.[6], it is claimed that the phase transition becomes so slow that it is impossible to produce the required density perturbations after inflation with normal parameter values. However, we find that the phase transition is fast because of the huge number of the destabilized Kaluza-Klein modes at the end of inflation. The number of the destabilized Kaluza-Klein modes becomes up to about $O\left(M_{p}^{2} / M_{*}^{2}\right)$. Although the problem of the slow phase transition seems to be solved by the Kaluza-Klein modes, another problem is induced by the excited Kaluza-Klein modes. The overproduction of the excited Kaluza-Klein modes is the problem, because they efficiently emit Kaluza-Klein gravitons when they decay into lower excited modes[5].

\section{Hybrid inflation due to bulk scalar fields}

In ref. [5], it is argued that a single field inflationary model cannot provide adequate density perturbations, either with the inflaton on the brane or with the bulk field inflation. Thus it is natural to invoke a hybrid inflationary model in higher dimensions with the potential for the bulk field,

$$
V\left(\phi_{5}, \sigma_{5}\right)_{5 D}=\lambda^{2} M_{*}\left(\sigma_{5 o}^{2}-\frac{1}{M_{*}} \sigma_{5}^{2}\right)^{2}+\frac{m_{\phi}^{2}}{2} \phi_{5}^{2}+\frac{g^{2}}{M_{*}} \sigma_{5}^{2} \phi_{5}^{2},
$$

where $\phi_{5}$ is the inflaton field with a chaotic initial condition. The coupling constants $\lambda$ and $g$ are assumed to be $O(1)$. To keep things as simple as possible, here we have assumed five dimensional theory with only one extra dimension. Extensions to higher dimensional

\footnotetext{
${ }^{2}$ In ref. 7 , interesting aspects of bulk inflation after the stabilization of the radion field is studied by using the KK modes in the 4D effective theory. See also ref. [8].
} 
theories are straightforward. As the higher dimensional fields $\phi_{5}$ and $\sigma_{5}$ has a mass of dimension $3 / 2$, the interaction terms are nonrenormalizable. Higher dimensional fields $\phi_{5}, \sigma_{5}$ are related to the effective four dimensional fields $\phi, \sigma$ by a scaling

$$
\phi=\sqrt{R} \phi_{5}, \sigma=\sqrt{R} \sigma_{5}
$$

which leads to couplings suppressed by the four dimensional Planck mass $M_{p}$, where $M_{p}^{2}=M_{*}^{3} R$. Here $R$ denotes the volume of the extra dimensions. After dimensional reduction, the effective four dimensional potential for the 0 -modes reads;

$$
\begin{aligned}
V(\phi, \sigma)_{4 D} \sim & \lambda^{2} \frac{M_{*}^{2}}{M_{p}^{2}}\left(\frac{M_{p}^{2} \sigma_{0}^{2}}{M_{*}^{2}}-\sigma^{2}\right)^{2} \\
& +\frac{m_{\phi^{2}}}{2} \phi^{2}+g^{2}\left(\frac{M_{*}}{M_{p}}\right)^{2} \phi^{2} \sigma^{2} .
\end{aligned}
$$

Including the Kaluza-Klein modes, the potential becomes]

$$
\begin{aligned}
V\left(\phi_{n}, \sigma_{n}\right)_{K K} \sim & \lambda^{2} \frac{M_{*}^{2}}{M_{p}^{2}}\left(\frac{M_{p}^{2} \sigma_{0}^{2}}{M_{*}^{2}}-\left(\sigma^{2}+\sum_{n} \sigma_{n}^{2}\right)\right)^{2} \\
& +\frac{m_{\phi^{2}}}{2}\left(\phi^{2}+\sum_{n} \phi_{n}^{2}\right)+g^{2}\left(\frac{M_{*}}{M_{p}}\right)^{2}\left(\phi^{2}+\sum_{n} \phi_{n}^{2}\right)\left(\sigma^{2}+\sum_{n} \sigma_{n}^{2}\right) \\
& +\sum_{n} \frac{n^{2}}{R^{2}} \phi_{n}^{2}+\sum_{n} \frac{n^{2}}{R^{2}} \sigma_{n}
\end{aligned}
$$

Let us first ignore the Kaluza-Klein modes in the effective potential and discuss the hybrid bulk field inflation induced by the potential eq.(2.3). In ref. [5], the parameter values $\sigma_{o} \sim M_{*}=10^{5} \mathrm{GeV}, m_{\phi} \sim 10 \mathrm{GeV}$ were considered. With these values, the density perturbation

$$
\frac{\delta \rho}{\rho} \sim\left(\frac{g}{2 \lambda^{3 / 2}}\right) \times 10^{-5}
$$

were obtained. Then the reheating temperature is calculated within the perturbation theory. In ref. [5], the decay rates of the inflaton field are already discussed. For the decay of the inflaton field into two Higgs fields on the brane, the decay width becomes

$$
\Gamma_{H H} \sim \frac{M_{*}^{4}}{32 \pi M_{p}^{2} m_{\phi}} .
$$

3 We have assumed that the nonrenormalizable terms in the five dimensional potential (2.3) are obtained by integrating out massive modes. These massive modes, which mediate the interactions $\sigma_{5}^{4}$ or $\phi_{5}^{2} \sigma_{5}^{2}$, are assumed to have no Kaluza-Klein number. 
On the other hand, their decay into excited Kaluza-Klein modes are highly suppressed,

$$
\Gamma_{\phi \phi \rightarrow \phi_{n} \phi_{n}} \sim \lambda^{2} \frac{M_{*}^{2}}{M_{p}^{2}} .
$$

Thus it seems appropriate to calculate the reheating temperature only by the decay into Higgs fields on the brane. Then the reheating temperature becomes $\sim 100 \mathrm{MeV}$, which satisfies the requirement for the successful big bang nucleosynthesis, and at the same time solves the problem of overproduction of Kaluza-Klein gravitons.

In this model, however, a serious problem was reported in ref. [6]. The authors of ref. [6] claimed that the phase transition becomes extremely slow and it is impossible to reproduce the present Universe with the above parameter values. Let us first make a brief review of the argument. The slope of the potential (2.3) in the $\phi$ direction is given by

$$
\frac{d V}{d \phi}=\left[2 g^{2}\left(\frac{M_{*}}{M_{p}}\right)^{2} \sigma^{2}+m_{\phi}^{2}\right] \phi,
$$

and the inflaton $\phi$ rolls down the potential with the Hubble parameter

$$
H=\sqrt{\frac{8 \pi}{3}} \frac{\lambda \sigma_{0}^{2}}{M_{*}}
$$

The inflaton $\phi$ slowly rolls down the potential until it reaches the critical value

$$
\phi_{c}=\frac{\lambda}{g}\left(\frac{\sigma_{0} M_{p}}{M_{*}}\right)
$$

where the potential in the $\sigma$ direction is destabilized. The evolution of the inflaton $\phi$ is given by

$$
\phi=\phi_{c} \exp \left[-\frac{1}{\sqrt{24 \pi} \lambda}\left(\frac{m_{\phi}}{\sigma_{0}}\right)^{2} M_{*} t\right]
$$

where we take the time variable $t$ as $t=0$ at $\phi=\phi_{c}$. In the $\sigma$ direction, the slope of the potential (2.3) is

$$
\frac{d V}{d \sigma}=\left(\frac{M_{*}}{M_{p}}\right)^{2}\left[\lambda^{2} \sigma^{2}+2 g^{2}\left(\phi^{2}-\phi_{c}^{2}\right)\right] \sigma .
$$

At $t=0$, the initial value of $\sigma$ is expected to be about $\sigma_{i n i} \sim H \sim M_{*}$. The phase transition lasts until the first term in eq.(2.8) comes to dominate the evolution. It happens when

$$
2 g^{2}\left(\frac{M_{*}}{M_{p}}\right)^{2} \sigma^{2} \sim m_{\phi}^{2}
$$


where $\sigma$ becomes about $\sigma_{\text {end }} \sim 10^{14} \mathrm{GeV}$. Solving the equation for $\sigma$, one obtains large e-foldings $N_{e} \sim 10^{5}$. During this period, $\sigma$ evolves from $\sigma_{i n i} \sim 10^{5} \mathrm{GeV}$ to $\sigma_{\text {end }} \sim 10^{14} \mathrm{GeV}$.

However, the Kaluza-Klein modes are important in this case. Seeing the effective potential eq.(2.4), one can easily find that not only the 0 -mode $\sigma$ but also the KaluzaKlein modes $\sigma_{n}$ are destabilized to contribute the phase transition. The number of the destabilized modes at the end of inflation is estimated by the potential (2.4),

$$
N_{K K} \sim\left(R \lambda \sigma_{0}\right) \sim\left(\frac{M_{p}}{M_{*}}\right)^{2}
$$

In the presence of a large number of destabilized channels near $\sigma \simeq \sigma_{n} \simeq 0$, the phase transition becomes inevitably fast.

In the original calculation of ref. [6], the initial value of $\sigma$ is $\sigma_{i n i} \sim H \sim M_{*} \sim 10^{5} \mathrm{GeV}$, which is much smaller than the critical value $\sigma_{\text {end }}$ in eq.(2.13). On the other hand, in the case when $\sigma^{2}$ in eq.(2.8) is replaced by the sum of the huge number of the Kaluza-Klein modes, the condition (2.13) is already satisfied as soon as the phase transition starts at $\phi \sim \phi_{c}$. In this case, each Kaluza-Klein mode $\sigma_{n}$ may have the initial value of $\sigma_{n} \sim H$, if the mass is smaller than the Hubble parameter.

Although the problem of the slow phase transition is avoided by the huge numbers of the Kaluza-Klein modes, there arises another serious problem. As is discussed in ref. [5], the reheating after the bulk inflation is not a problem when only the 0-mode oscillates and decays after inflation. In this model, however, the decay products of the excited Kaluza-Klein modes will dominate the Universe after inflation, because the inflation ends with the oscillation and the production of the excited Kaluza-Klein modes. Then the Kaluza-Klein gravitons are produced when the excited Kaluza-Klein modes decay into lower modes. The decay rate is enhanced by the large number of accessible modes in the final state, thus the excited modes are very short lived[5].

Let us examine the condition for the Kaluza-Klein gravitons to decay safe before nucleosynthesis. The decay width of the Kaluza-Klein gravitons into fields on the brane is estimated in ref.[10],

$$
\Gamma \sim \frac{E^{3}}{M_{p}^{2}} .
$$

Here $E$ denotes the energy of the graviton propagating in the bulk. In the most optimistic case, when $E \sim M_{*}$, the Kaluza-Klein gravitons may decay before nucleosynthesis 
if the fundamental scale is larger than $10^{6} \mathrm{GeV}$.

\section{Conclusions and Discussions}

In this paper, we have discussed the possibility of successful hybrid inflation due to the bulk scalar field, in models with large extra dimensions. Bulk field inflation is already discussed in papers [5, 6], where the problem of Kaluza-Klein gravitons [5] and the problem of the slow phase transition [6] are discussed. In ref.[0], it is argued that the production rate of the Kaluza-Klein graviton is so small that the energy of the inflaton is safely drained into the standard model fields on the brane. However, including the KaluzaKlein interactions, the production of the Kaluza-Klein gravitons becomes efficient. The problem of the slow phase transition is discussed in ref.[6]. However, because of the huge number of excited Kaluza-Klein states that becomes unstable at the end of inflation, the phase transition becomes fast. The remaining problem is the overproduction of the Kaluza-Klein gravitons, which puts a lower bound to the fundamental scale. Even in the most optimistic case, the bound becomes $M_{*}>10^{6} \mathrm{GeV}$.

The significant effect of the Kaluza-Klein excited states, which we have discussed for the bulk inflation, is generically important in cosmological models that utilizes the phase transition of the bulk field.

\section{Acknowledgment}

We wish to thank K.Shima for encouragement, and our colleagues in Tokyo University for their kind hospitality.

\section{References}

[1] I.Antoniadis, N.A-Hamed, S.Dimopoulos, and G.R.Dvali, Phys.Lett.B436:257263,1998; I.Antoniadis, Phys.Lett.B246:377-384,1990; N.A-Hamed, S.Dimopoulos and G.R.Dvali, Phys.Lett.B429:263-272,1998. 
[2] G.R. Dvali, G. Gabadadze, Phys.Lett.B460:47-57,1999; T.Matsuda, Phys.Rev.D65:103501,2002, Phys.Rev.D65:103502,2002, Phys.Rev.D66:023508,2002, Phys.Rev.D65:107302,2002, hep-ph/0205331; A. Masiero, M. Peloso, L. Sorbo, and R. Tabbash, Phys.Rev.D62:063515,2000; A.Mazumdar, Nucl.Phys.B597(2001)561, Phys.Rev.D64(2001)027304; A. Mazumdar and A. Perez-Lorenzana, Phys.Rev.D65:107301,2002; R. Allahverdi, K. Enqvist, A. Mazumdar, and A. Perez-Lorenzana, Nucl.Phys.B618:277-300,2001; A.Pilaftsis, Phys.Rev.D60:105023,1999; R.Allahverdi, K.Enqvist, A.Mazumdar and A.PLorenzana, Nucl.Phys. B618:377,2001; S. Davidson, M. Losada, and A. Riotto, Phys.Rev.Lett.84:4284-4287,2000.

[3] N. Kaloper and A. D. Linde, Phys.Rev.D59:101303,1999; D. H. Lyth, Phys.Lett.B466:85-94,1999.

[4] N. Arkani-Hamed, S. Dimopoulos, N. Kaloper, and J. March-Russell, Nucl.Phys.B567:189-228,2000

[5] R. N. Mohapatra, A. Perez-Lorenzana, and C. A. de S. Pires, Phys.Rev.D62:105030,2000

[6] A. M. Green and A. Mazumdar, Phys.Rev.D65:105022,2002

[7] P. Kanti, K. A. Olive, Phys.Rev.D60:043502,1999; Phys.Lett.B464:192-198,1999.

[8] Yun-Song Piao, Wen-bin Lin, Xin-min Zhang, and Yuan-Zhong Zhang, Phys.Lett.B528:188-192,2002.

[9] G. R. Dvali and S.H.Henry Tye, Phys.Lett.B450:72-82,1999; G. R. Dvali, Phys.Lett.B459:489-496,1999;

[10] N. Arkani-Hamed, S. Dimopoulos, and G.R. Dvali, Phys.Rev.D59:086004,1999. 